\title{
Kurtzmaniella gen. nov. and description of the heterothallic, haplontic yeast species Kurtzmaniella cleridarum sp. nov., the teleomorph of Candida cleridarum
}

\author{
Marc-André Lachance ${ }^{1}$ and William T. Starmer ${ }^{2}$ \\ ${ }^{1}$ Department of Biology, University of Western Ontario, London, ON N6A 5B7, Canada \\ ${ }^{2}$ Department of Biology, Syracuse University, Syracuse, NY 13244, USA
}

Correspondence

Marc-André Lachance

lachance@uwo.ca

\begin{abstract}
The teleomorph of Candida cleridarum was discovered through the detection of conjugation between isolates of a large collection from the nitidulid beetles of the genus Carpophilus found in the flowers of various cacti in Arizona, USA. The previous oversight of the sexual cycle of this yeast is attributed to the inequality (ca. $5: 1$ ) of the two mating types. Extensive conjugation between compatible mating types is observed after overnight incubation on $5 \%$ malt agar, followed after 3-5 days by the formation of mature asci. The hat-shaped ascospores are reminiscent of those seen in Kodamaea species, which are members of the same guild. However, published analyses of D1/D2 large subunit rDNA sequences indicate an affinity with the genus Debaryomyces. As the latter is polyphyletic and morphologically heterogeneous, and in view of the distinct life cycle of the new teleomorph, the new genus Kurtzmaniella is described with a novel species, Kurtzmaniella cleridarum sp. nov. Given the close relatedness of Kurtzmaniella cleridarum sp. nov. to Candida quercitrusa, Candida oleophila and Candida railenensis, for which several natural isolates were available, strains of these species were mixed in pairs under the conditions found favourable for the former. Conjugation was not detected in those species. The type strain of Kurtzmaniella cleridarum sp. nov. is UWOPS 99-101.1 ${ }^{\top}\left(=\mathrm{CBS} 8793^{\top}=\mathrm{NRRL}\right.$ $\mathrm{Y}-48386^{\top}, h^{+}$), type of Candida cleridarum. The allotype is UWOPS 07-123.1 (=CBS $10688=$ NRRL $Y-48387, h^{-}$).
\end{abstract}

Candida cleridarum was described by Lachance et al. (2001) from a collection of strains recovered from flowers of cacti of the deserts of Arizona and California, USA, or from beetles that occur in the flowers. The species was named after the clerid beetles found in some of the flowers, but it was later found (Lachance \& Bowles, 2002) that the major vector of yeasts in these flowers is the nitidulid genus Carpophilus. Carpophilus pallipennis is known to occur consistently in the flowers of Opuntia and several other cactus genera of that region (Grant \& Connell, 1979), whereas flowers of the sympatric saguaro (Carnegiea gigantea) harbour Carpophilus longiventris (Werner, 1959). Nitidulid (sap) beetles found in the corolla of ephemeral flowers carry yeasts that are often highly specific to the beetle species (Lachance et al., 2003a). The nature of the association is not known, but one of the

Abbreviation: CTAB, cetyldimethylethylammonium bromide.

The GenBank/EMBL/DDBJ accession number for the gene sequence of the D1/D2 variable domains of the large subunit rDNA of Kurtzmaniella cleridarum sp. nov. is AF251552.

The Mycobank accession number for Kurtzmaniella cleridarum sp. nov. is MB511088 (http://www.mycobank.org). reasons for the specificity may well be the volatile substances released by yeasts as they ferment plant sugars. Yeast fermentation products can act as strong attractants for Carpophilus species that are pests of certain crop plants (Nout \& Bartelt, 1998) and may be important in preserving beetle-yeast associations.

In three previous collections of yeasts found in cactus flowers (Lachance et al., 2001; Lachance \& Bowles, 2002; and unpublished data), Candida cleridarum was clearly dominant across the entire collection area, which included sites in southern Arizona and southern California. The exception was a few samples collected in two localities near Phoenix, Arizona, where a small number of isolates of the endemic species Metschnikowia arizonensis were also found. With the objective of gaining new insights into the basis of these distributions, we recently made an intense collection of the yeasts associated with Carpophilus spp. in the flowers of cacti in five Arizona localities. Of the 107 yeast strains recovered, 102 were identified as Candida cleridarum based on morphology and growth responses. Only one of the remaining strains was identified as $M$. arizonensis. However, we were successful in detecting conjugation 
among strains of Candida cleridarum, which led us to show, through mating experiments, that they belong to a single ascosporic species.

\section{Isolation of conjugating strains}

Table 1 details the origin of recent isolates plus ten isolates that were retained from a collection conducted in 1999 (Lachance et al., 2001). The sites of the recent collection were visited in April 2007, at which time flowering was extensive in flat and cylindrical Opuntia species as well as Echinocereus species. Insects were removed aseptically from flowers and were allowed to walk for $10-20 \mathrm{~min}$ on the surface of agar plates. The yeasts deposited on the agar surface were then spread with an inoculation loop. To maximize the probability of detecting mating types of $M$. arizonensis, insects were allowed to walk on two different agar media. As a general isolation medium, we used YM agar $(1.0 \%$ glucose, $0.5 \%$ peptone, $0.3 \%$ malt extract, $0.3 \%$ yeast extract and $2.0 \%$ agar) supplemented with $100 \mathrm{mg}$ chloramphenicol $\mathrm{l}^{-1}$. The second medium additionally contained $\left(1^{-1}\right), \quad 10 \mathrm{mg}$ cetyldimethylethylammonium bromide (CTAB) and $5 \mathrm{mg}$ phloxine $\mathrm{B}$. $\mathrm{CTAB}$, a toxic detergent, has been used in this laboratory as a selective agent for species of the genus Metschnikowia (Lachance et al., 2003a). It happens that Candida cleridarum is also resistant to CTAB (Lachance et al., 2001). Phloxine B is a vital stain that has been used, mostly by geneticists, for the detection of colonies formed by physiological variants (Nagai, 1963). It would seem that the dye preferentially penetrates cells that have entered $G_{1}$ arrest. We have found it useful, in previous studies, for the on-site detection of interacting mating types of heterothallic Metschnikowia species on plates inoculated with floricolous beetle yeasts. When two Metschnikowia sp.

Table 1. Origin and mating types of isolates of Kurtzmaniella cleridarum sp. nov.

The 1999 collection has been described in detail by Lachance et al. (2001).

\begin{tabular}{|lrrr|}
\hline \multirow{2}{*}{ Locality } & Year & \multicolumn{2}{c|}{ Mating type } \\
\cline { 2 - 5 } & & $\boldsymbol{h}^{+}$ & $\boldsymbol{h}^{-}$ \\
\hline Lost Dutchman State Park & 1999 & 2 & \\
Lost Dutchman State Park & 2007 & 19 & 7 \\
Pinal Highway & 1999 & 2 & \\
Boyce Thompson Arboretum & 2007 & 4 & 2 \\
Saguaro National Park East & 2007 & 19 & 2 \\
Saguaro National Park West & 2007 & 20 & 2 \\
Gilbert Ray Campground & 1999 & 2 & \\
Brown Canyon, Coronado National Forest & 2007 & 11 & 3 \\
Organ Pipe Cactus National Monument & 1999 & 1 & \\
Organ Pipe Cactus National Monument & 2007 & 11 & 2 \\
Joshua Tree National Park, California & 1999 & 1 & 1 \\
Amboy, California & 1999 & 1 & \\
Total & & 93 & 19 \\
\hline
\end{tabular}

colonies of complementary mating types grow in contact with each other, one of the colonies often picks up and concentrates the red colour. In the present study, we noticed that some confluent colonies of Candida cleridarum formed an intensely coloured zone at the point of contact. We later confirmed that the dye was concentrated at the point where cells were conjugating, although microscopic examination showed that the conjugants themselves did not absorb significant quantities of the dye, in contrast to dead cells, which appeared bright red.

\section{Mating type distribution}

The mating type $h^{+}$, arbitrarily assigned to the type strain of Candida cleridarum UWOPS 99-101.1 ${ }^{\mathrm{T}}$, predominated in all localities with a fivefold excess over mating type $h^{-}$. Of the strains listed in the original description of Candida cleridarum (Lachance et al., 2001), ten were preserved in the UWOPS culture collection. Of these, only one (UWOPS 99-117.1) had the mating type $h^{-}$(Table 1). This explains in part why conjugation had been overlooked in previous collections. Another reason was the choice of mating medium. When examining mixtures of strains for possible conjugation and ascus formation, we normally use, by default, YCBAS agar (yeast carbon base, Difco, with $0.01 \%$ ammonium sulphate). This medium has generally given excellent results with heterothallic, haplontic species of the genera Clavispora, Metschnikowia, Kodamaea, Starmerella and Wickerhamiella, although there have been exceptions (Lachance et al., 2000). In the present case, given that the interactions were detected from the accumulation of phloxine B occurred on YM agar, a rich medium, we compared the efficacy of several media, including YCBAS, YM, 20-fold diluted V8 and $1 \%$ and $5 \%$ malt agar. The last medium gave markedly better results and YCBAS agar was particularly poor.

\section{Characterization of new isolates}

The growth characteristics of the yeasts were determined following standard methods (Yarrow, 1998). The isolates identified as Candida cleridarum matched the original description well, with some minor variations that may represent artefacts of media preparation. Specifically, most strains gave a slow and weak reaction for gelatin hydrolysis, which was previously reported as negative. Two strains that were picked on the basis of the formation of smaller colonies on the medium containing CTAB had slightly different growth patterns. Strain UWOPS 07-101a1 $\left(h^{+}\right)$ exhibited no growth at all on sucrose, maltose or melezitose, although other growth responses were normal. Strain UWOPS 07-131.2 $\left(h^{+}\right)$, grew weakly on most media. Growth on citrate varied markedly across all strains.

\section{Kurtzmaniella gen. nov.}

The discovery of ascus formation in Candida cleridarum requires assignment to a teleomorphic genus. The current 
practice is to attempt the maintenance of genera that are monophyletic as inferred by DNA sequence analyses, as well as morphologically consistent. The present case is difficult in this respect. The closest relative of Candida cleridarum based on D1/D2 large subunit rDNA sequences is Candida fragi, as shown in Fig. 1. This and other analyses (Kurtzman \& Robnett, 1998; Lachance et al., 2001; Kurtzman et al., 2001) showed that these species form a clade with a few other Candida species whose closest known ascosporic relatives are in the genus Debaryomyces. However, the latter genus has representatives in three clades. The clade nearest to Candida cleridarum and close relatives comprises, among others, Debaryomyces hansenii, which forms automictic asci with usually a single roughwalled ascospore, and Debaryomyces (Wingea) robertsii, with characteristic lenticular ascospores that come singly or in pairs. The second nearest clade contains automictic species that include Debaryomyces (Schwanniomyces) occidentalis, with mostly a single rugose, ledged ascospore, and Debaryomyces polymorphus, with two or more smooth, spheroidal ascospores. A third, more distant clade does not form a monophyletic set with the others and contains a mixture of Debaryomyces species and certain Pichia species that form spheroidal ascospores without a ledge. A multigene study in progress (C. P. Kurtzman, personal communication) confirms this phylogenetic structure. None of the known species of the genus Debaryomyces produce pairs of hat-shaped ascospores by conjugation of heterothallic strains. In view of all the above considerations, we think it justified to erect a separate genus to accommodate the teleomorph of Candida cleridarum and eventually those of neighbouring species. We propose to

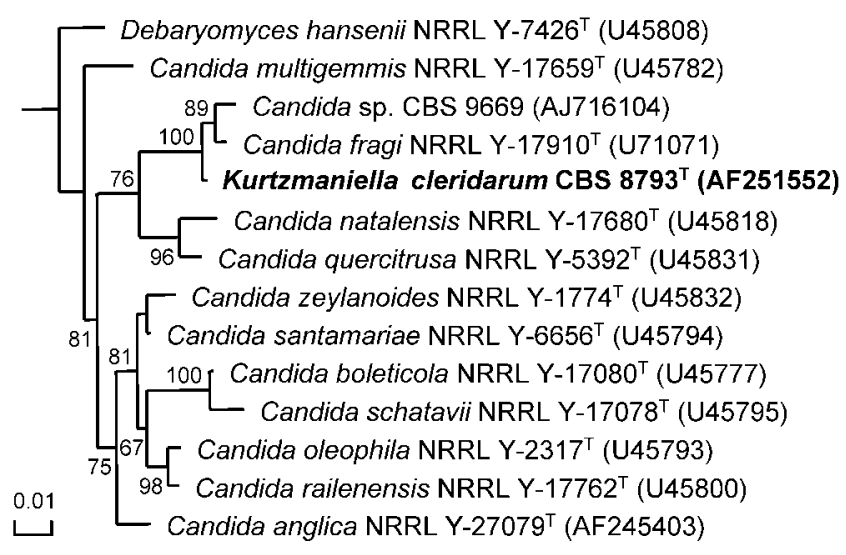

Fig. 1. Neighbour-joining phylogram of Kurtzmaniella cleridarum sp. nov. and related species based on the D1/D2 variable domains of the large subunit rDNA. Debaryomyces hansenii represents the sister clades. The tree was rooted by the addition of an outgroup sequence (Candida glucosophila NRRL Y $-17781^{\top}$, GenBank accession no. U45849, not shown). Bootstrap values of $50 \%$ or more are shown. Bar, 0.01 sequence divergence $\left(K_{\text {nuc }}\right)$. The DNA sequences were retrieved from GenBank and analysed with the DNAMAN program (Lynnon Corporation). name this genus Kurtzmaniella gen. nov., in honour of Cletus P. Kurtzman, in recognition of his innumerable contributions to yeast systematics. Most noteworthy are Kurtzman's leadership in the work that led to the first and still only operational molecular barcoding system for yeast identification and his relentless efforts to provide phylogenetic circumscription of yeast genera through multi-gene sequence analysis.

\section{Search for other Kurtzmaniella species}

In view of the close relatedness of Kurtzmaniella cleridarum sp. nov. to Candida quercitrusa, Candida oleophila and Candida railenensis (Fig. 1), for which several natural isolates were available in our collection, strains of these species, including the type cultures, were mixed in every possible pair under the conditions found favourable for $K$. cleridarum. Conjugation was not detected for 13 strains of Candida oleophila, 9 strains of Candida quercitrusa or 12 strains of Candida railenensis. Although Candida fragi is known from only a single strain, it differs from $K$. cleridarum by only five substitutions, each in the ITS $/ 5.8 \mathrm{~S}$ (GenBank accession nos AY344066 and AY344065) and the D1/D2 LSU (U71071 and AF251552) rDNA sequences, respectively, leaving open the possibility that the two taxa might represent variants of a single polymorphic species (Lachance et al., 2003b). The type strain of Candida fragi (CBS $7702^{\mathrm{T}}$ ) was therefore mixed with the two mating types of $\mathrm{K}$. cleridarum and examined for signs of a mating reaction. No interactions of any kind were detected. Furthermore, profound differences between the two species at the cellular and colony level were noted. $K$. cleridarum forms larger, mostly ovoidal cells and smooth colonies in contrast to Candida fragi where the cells are smaller, elongate, and angular, with dull and rugose colonies.

The Vienna edition of the International Code of Botanical Nomenclature (McNeill, 2006) now allows the epitypification of species in an anamorphic genus if a teleomorph is discovered for one species in the genus. However, it is not clear how the relevant Article (59.7) would apply in the present circumstance, as the species most closely related to $K$. cleridarum are part of a large polyphyletic genus (Candida) in which most species are not closely related. In the absence of objective evidence such as interspecific mating, the transfer of neighbouring species to Kurtzmaniella would require making a typological decision as to which additional species to include: only Candida fragi or other species in neighbouring subclades. This would run the risk that new information would cause the later transfer of some of the species to yet another genus, an option that we do not view favourably.

\section{Ecology and biogeography}

Lachance et al. (2001) commented on the extraordinary habitat specificity of Candida cleridarum. The isolation of over a hundred more representatives confirms this 
remarkable distribution. In addition, the fact that each of the five sites sampled yielded both mating types in approximately similar $(5: 1)$ ratios and the absence of any local peculiarities in growth responses (i.e. the aforementioned variation in citrate utilization is randomly distributed) suggest that the species may constitute a panmictic population across its known range. Further studies will be required to test this hypothesis. We also hope to study mating type inheritance to see if the evolutionary fitness of $h^{-}$strains might be less that that of $h^{+}$strains.

\section{Physiological convergence in the floricolous beetle yeast guild}

Another striking feature emerging from our studies of yeasts associated with floricolous beetles is the similarity of the dominant species in their growth responses, irrespective of phylogenetic affinity. The nutritional profile of Kurtzmaniella cleridarum is remarkably similar to that of many guild members that belong to the Metschnikowia and Kodamaea clades. Specifically, these yeasts have a very strong propensity for assimilating sucrose, galactose, maltose, melezitose, sorbose, xylose, mannitol, glucitol, succinic and citric acids, 2-keto-D-gluconic acid and $\mathrm{N}$ acetyl-D-glucosamine, but not L-rhamnose or D-ribose. Suitable nitrogen sources include ethylamine, cadaverine and usually lysine, but not nitrate or nitrite. Tween 80 is often hydrolysed. All species are fermentative and sensitive to cycloheximide $\left(100 \mathrm{mg} \mathrm{l}^{-1}\right)$ and most are resistant to CTAB (10 $\mathrm{mg} \mathrm{l}^{-1}$ or more). Whereas the possession of any one of these features is far from exceptional, the convergence of so many characteristics is improbable and suggests that these traits might jointly define the fundamental niche of floricolous beetle-associated yeasts.

\section{Latin diagnosis of Kurtzmaniella gen. nov.}

Multiplicatio vegetativa gemmatione multilaterali. Cellulae ovoidae ad ellipsoidae. Pseudomycelium potest. Asci gemini. Ascosporae binae globosae compressae, petasiformae, ad maturitatem liberantur.

Fermentatio fieri potest. Ad Saccharomycetaceae pertinens. Species typica: Kurtzmaniella cleridarum Lachance \& Starmer sp. nov.

\section{Description of Kurtzmaniella gen. nov.}

Kurtzmaniella (Kurtz.man.i.el'la. N.L. nom. f. n. Kurtzmaniella named in honour of Cletus P. Kurtzman, in recognition of his major contributions to yeast systematics).

The genus belongs to the family Saccharomycetaceae. Vegetative reproduction is by multilateral budding. Vegetative cells are ovoid to ellipsoidal. Asci are conjugated and form usually two flattened, hat-shaped ascospores with a conspicuous basal ledge. Asci are slowly evanescent. Rudimentary pseudomycelia are formed. The single species is fermentative. Type species: Kurtzmaniella cleridarum Lachance \& Starmer sp. nov. The name is registered under Mycobank number MB511085.

\section{Latin diagnosis of Kurtzmaniella cleridarum sp. nov.}

In medio liquido post dies tres cellulae singulae, binae, aut in catenis brevis, ovoidae aut bacilliformes $(3-5 \times 3-8 \mu \mathrm{m})$. Cellulae longiores formari possunt. Post unum mensem annulus tenuis et sedimentum formantur. Cultura in agaro malti post dies $14\left(17{ }^{\circ} \mathrm{C}\right)$, infimo-convexa, tumulosa, glabra, candida et butyrosa. In agaro farinae Zea mays post dies 14 pseudomycelium formatur. Asci post conjugationem stirpium convenientium formantur. Glucosum et sucrosum (exigue et lente) fermentantur. Sucrosum, galactosum (lente), maltosum, melezitosum, methyl- $\alpha$-D-glucosidum (exigue), salicinum, L-sorbosum, D-xylosum, D-ribosum (variabile et exigue), ethanolum (exigue), glycerolum, ribitolum (lente), xylitolum (lente), mannitolum, glucitolum, acidum lacticum (exigue), acidum succinicum, acidum citricum (lente et variabile), acidum malicum (lente), acidum gluconicum (lente), glucono- $\Delta$-lactonum (lente), 2-keto-D-gluconatum, D-glucosaminum (exigue), $N$-acetyl-D-glucosaminum et hexadecanum (exigue) assimilantur, at non inulinum, raffinosum, melibiosum, lactosum, trehalosum (aliquando exigue), amylum solubile, cellobiosum L-rhamnosum, L-arabinosum, D-arabinosum, methanolum, 1-propanolum, 2-propanolum, 1-butanolum, erythritolum, galactitolum, meso-inositolum, acetonum nec ethyl acetas. Ethylaminum, lysinum et cadaverinum assimilantur at non natrium nitricum nec natrium nitrosum. Ad crescentiam vitamina externa necessaria sunt. Augmentum in $37{ }^{\circ} \mathrm{C}$. Habitat floras cactorum et coleopteros junctes generi Carpophilus in Arizona et California. Typus UWOPS 99-101.1 ${ }^{\mathrm{T}}$. Allotypus UWOPS 07-123.1. In collectione zymotica Centraalbureau voor Schimmelcultures, Trajectum ad Rhenum, sub no. CBS $8793^{\mathrm{T}}$ et CBS 10688 depositae sunt.

\section{Description of Kurtzmaniella cleridarum sp. nov.}

Kurtzmaniella cleridarum (cle.ri.da' rum. N.L. gen. pl. fem. n. cleridarum of Cleridae, referring to the family of beetles that was originally thought to act as vectors for this yeast species).

The description is identical to that given for Candida cleridarum by Lachance et al. (2001) except for the following variances. Conjugation between haploid strains of compatible mating types is observed after 1 day at $25{ }^{\circ} \mathrm{C}$. Mature asci containing usually two hat shaped ascospores, $2-2.5 \times 3-4 \mu \mathrm{m}$ occur after $3-5$ days (Fig. 2). The lobe containing the ascospores gradually deliquesces over several more days. Ascospore formation is abundant on $5 \%$ malt extract agar, weak on $1 \%$ malt or 20 -fold dilute V8 agar, and rare or absent on YCBAS. After 3 weeks on malt gelatin, liquefaction is weak to moderate. The habitat 


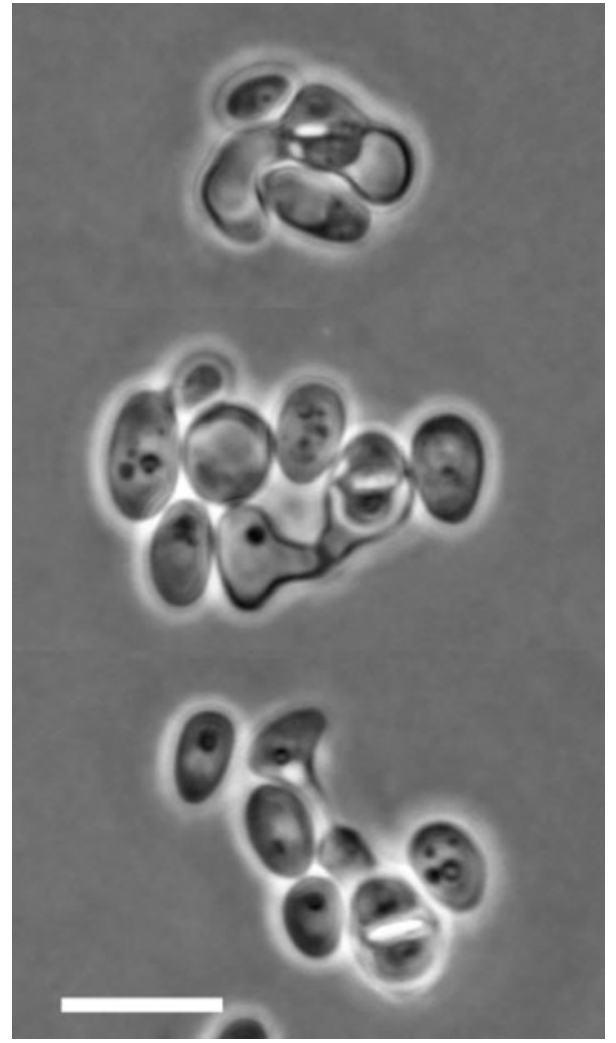

Fig. 2. Phase-contrast micrographs of asci of Kurtzmaniella cleridarum sp. nov. after 4 days at $25{ }^{\circ} \mathrm{C}$ on $5 \%$ malt agar. Bar, $10 \mu \mathrm{m}$.

is in flowers of cacti and associated beetles of the genus Carpophilus in the south-west United States.

The type strain of Kurtzmaniella cleridarum is strain UWOPS 99-101.1 ${ }^{\mathrm{T}}$, the type of Candida cleridarum. It was isolated from a beetle collected from a flower of Opuntia phaeacantha in Lost Dutchman State Park, Arizona, USA. The designated allotype is strain UWOPS 07-123.1 recovered from a specimen of Carpophilus sp. found in a flower of Opuntia engelmanii, in Brown Canyon, Coronado National Forest, Arizona, USA. The strains have been deposited in the collection of the Yeast Division of the Centraalbureau voor Schimmelcultures, Utrecht, the Netherlands, as strains CBS $8793^{\mathrm{T}}$ (=NRRL Y-48386 ${ }^{\mathrm{T}}$ ) and CBS 10688 (=NRRL Y-48387). The binomial is registered under Mycobank number MB511088.

\section{Acknowledgements}

This work was funded by the Natural Science and Engineering Research Council of Canada (M.-A. L.) and by the National Science Foundation, USA (W.T.S.). We are grateful to various park authorities for permission to collect. We thank Randi Starmer, Dan Austin and Adam Bjork for field assistance, TaeEun Ahn, Joonyoung Ji and Alison Wardlaw for laboratory assistance, and Clete Kurtzman for sharing unpublished data and commenting on the manuscript.

\section{References}

Grant, V. \& Connell, W. A. (1979). The association between Carpophilus beetles and cactus flowers. Plant Syst Evol 133, 99-102.

Kurtzman, C. P. \& Robnett, C. J. (1998). Identification and phylogeny of ascomycetous yeasts from analysis of nuclear large subunit (26S) ribosomal DNA partial sequences. Antonie van Leeuwenhoek 73, 331-371.

Kurtzman, C. P., Robnett, C. J. \& Yarrow, D. (2001). Three new species of Candida from apple cider: C. anglica, C. cidri, and C. pomicola. Antonie van Leeuwenhoek 80, 237-244.

Lachance, M. A. \& Bowles, J. M. (2002). Metschnikowia arizonensis and Metschnikowia dekortorum, two new large-spored yeast species associated with floricolous beetles. FEMS Yeast Res 2, 81-86.

Lachance, M. A., Bowles, J. M., Mueller, C. \& Starmer, W. T. (2000). On the biogeography of yeasts in the Wickerhamiella clade and description of Wickerhamiella lipophila sp. nov., the teleomorph of Candida lipophila. Can J Microbiol 46, 1145-1148.

Lachance, M. A., Bowles, J. M., Chavarria Diaz, M. M. \& Janzen, D. H. (2001). Candida cleridarum, Candida tilneyi and Candida powellii, three new yeast species isolated from insects associated with flowers. Int J Syst Evol Microbiol 51, 1201-1207.

Lachance, M. A., Bowles, J. M. \& Starmer, W. T. (2003a). Geography and niche occupancy as determinants of yeast biodiversity: the yeastinsect-morning glory ecosystem of Kīpuka Puaulu, Hawai'i. FEMS Yeast Res 4, 105-111.

Lachance, M. A., Daniel, H. M., Meyer, W., Prasad, G. S., Gautam, S. P. \& Boundy-Mills, K. (2003b). The D1/D2 domain of the large-subunit rDNA of the yeast species Clavispora lusitaniae is unusually polymorphic. FEMS Yeast Res 4, 253-258.

McNeill, J. (2006). International Code of Botanical Nomenclature (Vienna Code), 569 pp. Ruggell, Lichtenstein: A. R. G. Gantner Verlag KG.

Nagai, S. (1963). Diagnostic color differentiation plates for hereditary respiration deficiency in yeast. J Bacteriol 86, 299-302.

Nout, M. J. R. \& Bartelt, R. J. (1998). Attraction of a flying nitidulid (Carpophilus humeralis) to volatiles produced by yeasts grown on sweet corn and a corn-based medium. J Chem Ecol 24, 1217-1239.

Werner, F. G. (1959). Carpophilus longiventris in saguaro blossoms (Coleoptera: Nitidulidae). Psyche (Stuttg) 66, 35-36.

Yarrow, D. (1998). Methods for the isolation and identification of yeasts. In The Yeasts, a Taxonomic Study, 4th edn, pp. 77-100. Edited by C. P. Kurtzman \& J. W. Fell. Amsterdam: Elsevier. 